\title{
FDTD Analysis of EM Field Effected by the Motion of Source and Vehicle
}

\author{
S. Sahrani ${ }^{1} \quad$ T. Akata $^{2} \quad$ M. Kuroda ${ }^{2}$
}

\begin{abstract}
A numerical technique for the analysis of the electromagnetic (EM) fields by moving sources and moving bodies can be significantly important for the realization of next generation nano-electronic devices particularly for mobile communication system. However, most of the analysis were primarily focused on the stationary source. We have previously proposed the Overset Grid Generation method combined with the FDTD method for the analysis of the EM field with moving body. In this paper, this technique is applied for the analysis of the EM field in a street cross section from moving vehicle and moving source. Here, the Doppler effect is observed by the moving vehicle and moving source.
\end{abstract}

\section{INTRODUCTION}

The futures of some engineering applications crucially depend on the advanced algorithm in the numerical modeling technique with powerful computational capabilities [1]-[5]. This paper describes a new numerical technique by Overset Grid Generation method combined with Finite-Difference TimeDomain (FDTD) method and Lorentz transformation to solve the electromagnetic (EM) field with moving boundaries. This numerical technique has been previously proposed for a linearly moving body and in rotating environment for higher velocity cases[6]-[8].

The FDTD method is widely used to solve the EM field problems due to its low computational complexity [9]. It is very easy to implement in any computing environment including on the structured grids suchlike the Overset Grid Generation method [10]. The overset grid generation method consists of main mesh and sub-mesh that overlapped each another. It can reduce the geometrically complex problem to a simple set of grids.

In this paper, the sub-mesh is assumed to move at high velocities considering Lorentz transformation [11]. Based on the central difference equation in FDTD method, the same space and time increment are required in each calculation. Here, the time components that were changed in Lorentz transformation are fixed by using linear interpolation scheme in the Overset Grid Generation method. This allows a coherent point in time component with the FDTD method, which is an important element of this proposed numerical technique. The proposed Overset Grid Generation method combined with the FDTD method and Lorentz transformation is applied to the analysis of the EM field when the input source is moving towards the moving body. Here, the parallel processing technique, Open MP is used to reduce the computing time. Based on the parallel implementation, the accuracy of this technique is verified. It is then applied for the analysis of the EM field at the intersection when both of the source and the vehicle are moving. The numerical results of the moving case are compared with the stationary case.

\section{COMPUTATIONAL ALGORITHM OF FDTD METHOD AND OVERSET GRID GENERATION METHOD}

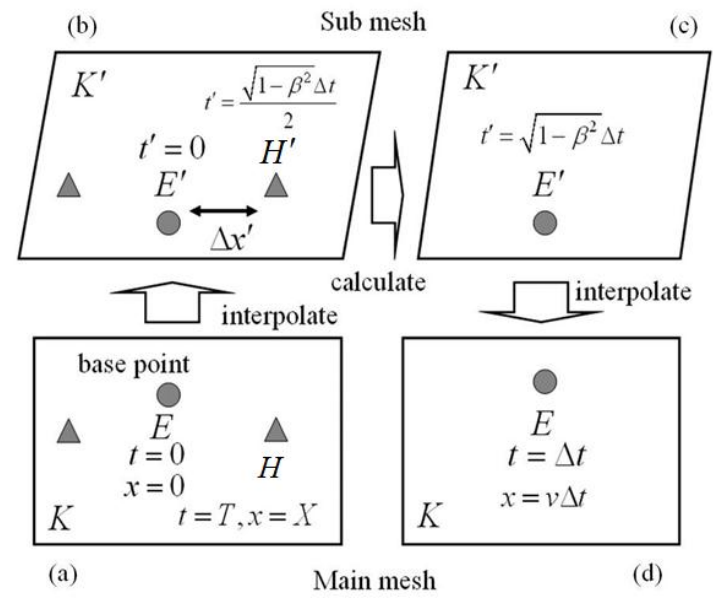

Fig. 1. Time and space algorithm for FDTD and Lorentz transformation by overset grid generation method.

Fig. 1 shows the diagram of the proposed computational algorithm based on the time and the space calculation in FDTD method and Lorentz transformation. Initially, the position of the sub-mesh is identified. Then, the EM field components on the main mesh are interpolated to the field components on the moving sub-mesh by applying Lorentz transformation[12],[13]. Here, the electric field is calculated in both meshes by the FDTD method. The calculated value of the electric field at the sub-mesh is interpolated back to the main mesh through Lorentz transformation. Here, the half time increment is advanced. At time $t=\Delta t$ in the main mesh, the value for time component on the moving sub-mesh in Fig. 1(c) should be:

\footnotetext{
${ }^{1}$ Faculty of Engineering, Universiti Malaysia Sarawak, Kota Samarahan, Malaysia

e-mail: sshafrida@feng.unimas.my

${ }^{2}$ School of Computer Science, Tokyo University of Technology, Katakura, Hachioji, Tokyo, Japan,

e-mail: akata.tatsuya@gmail.com

${ }^{2}$ School of Computer Science, Tokyo University of Technology, Katakura, Hachioji, Tokyo, Japan,

e-mail:kuroda@stf.teu.ac.jp
} 